\title{
WAS WisSen WIR ÜBER die GeflüChTETEN, DIE 2015 ZU UNS KAMEN?
}

Isabella BUBER-ENNSER, Vienna Institute of Demography, Österreichische Akademie der Wissenschaften, Wien

Judith Kohlenberger, Institut für Sozialpolitik, Department für Sozioökonomie, Wirtschaftsuniversität Wien

Bernhard RENGS, Vienna Institute of Demography, Österreichische Akademie der Wissenschaften, Wien

\section{INHALT}

1 Einleitung 109

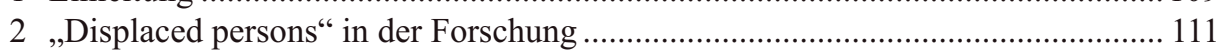

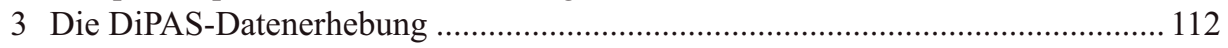

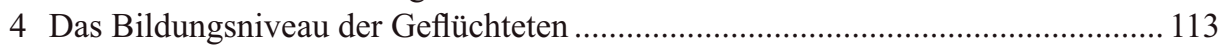

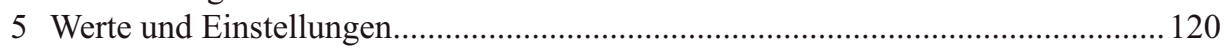

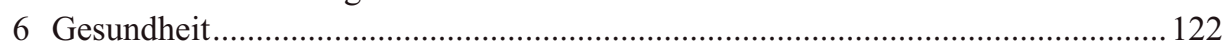

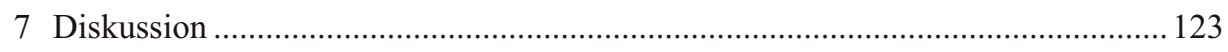

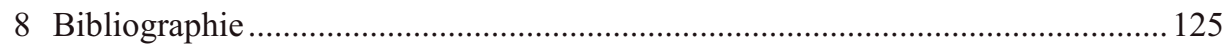

\section{Einleitung}

Österreich hatte traditionell hohe Zahlen von Asylsuchenden, was sich aus seiner geographischen Lage, dem historischen Hintergrund des Habsburgerreichs und den politischen Unruhen der jüngeren Zeitgeschichte in den östlichen Nachbarländern (z.B. Ungarn, Ex-Tschechoslowakei, Ex-Jugoslawien) erklärt (FAssmann \& STACHER 2003; Franz 2005; Halilovich 2013; UNHCR 2011) (vgl. Abb. 1). In den vergangenen Jahrzehnten war Österreich ein bedeutendes Aufnahmeland (HALILOvich 2013), obwohl die gängige Politik hinsichtlich der Gewährung von Asyl und des Zugangs zum Arbeits- 
markt tendenziell eher restriktiv war (RosenBERGER \& KöNIG 2012). Während die überwiegende Mehrheit der im Jahr 2015 nach Europa Geflüchteten Asyl in Deutschland beantragte (wo im Jahr 2015 fast eine halbe Million Asylsuchende einreisten, BAMF 2016a), kam ein beträchtlicher Anteil auch nach Österreich: Insgesamt stellten hier 88.098 Personen einen Asylantrag (BMI 2016). Diese Zahl entspricht etwa einem Prozent der österreichischen Bevölkerung und knapp 7\% aller Asylwerber/innen in der EU im Jahr 2015, was Österreich zum viertgrößten Zielland machte (EUROSTAT 2016). Die meisten Asylanträge wurden zwischen Sommer und Herbst 2015 gestellt (60.000 zwischen Juli und Dezember).

\section{Abb. 1: Asylanträge in Österreich}

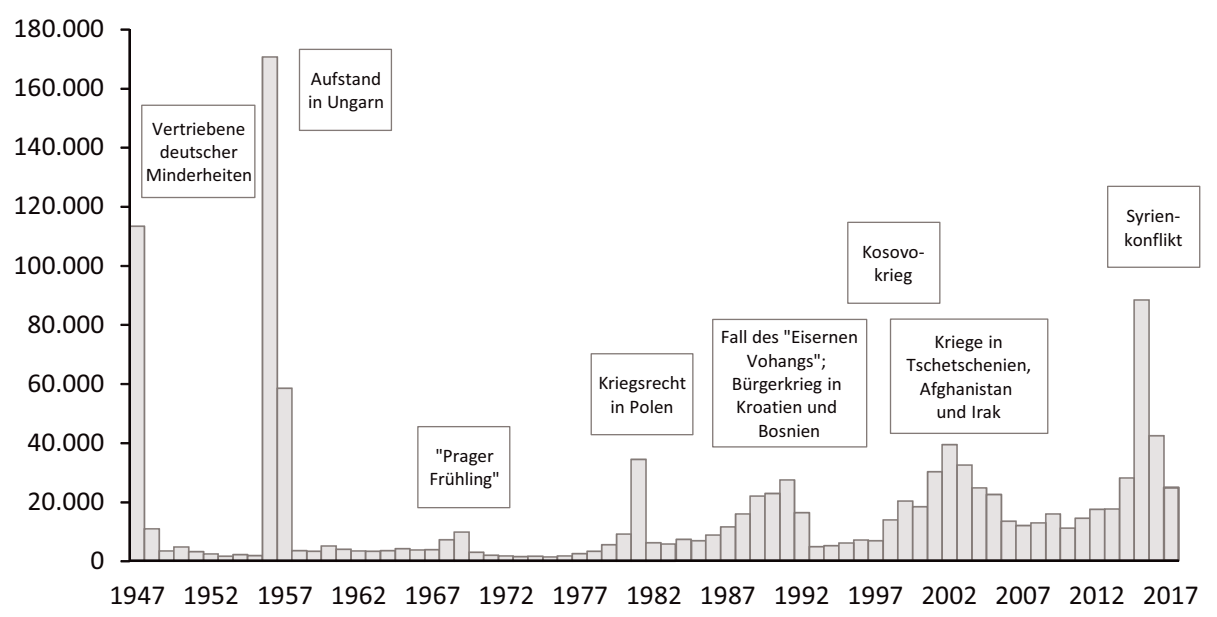

Quellen: Marik-Lebek \& Wisbauer (2017), BMi (2018), Statistics Austria (2018), UNHCR (2013b).

Die Staatsbürgerschaft der Asylsuchenden variierte sowohl zeitlich als auch örtlich innerhalb der europäischen Aufnahmeländer (BAMF 2016b; BMI 2016; EJPD 2016; Fargues 2015; Migrationsverket 2016; UDI 2016b). Die meisten Asylantragstellenden im Jahr 2015 in Österreich kamen aus Syrien, Afghanistan und dem Irak (71\%). Auch in Deutschland, Schweden, den Niederlanden, Finnland und Norwegen, die im Jahr 2015 ebenfalls viele Geflüchtete aufnahmen, kam ein Großteil der Asylsuchenden aus den drei genannten Ländern (vgl. Abb. 2). 
Abb. 2: Nationalitäten der Asylantragsteller/innen im Jahr 2015 im europäischen Vergleich

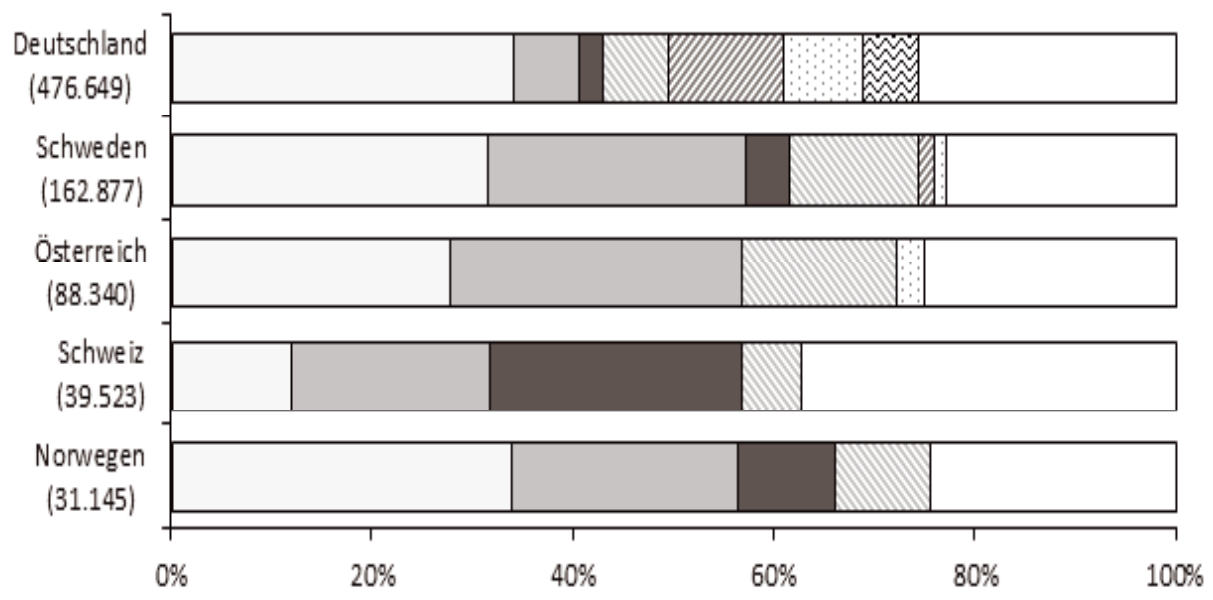

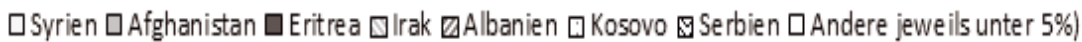

Quellen: BMI (2016), BAMF (2016b), EJPD (2016), UDI (2016a), MigRATIONSVERKET (2016).

Angesichts der gesellschaftlichen Bedeutung der großen Zahl von Geflüchteten, die im Jahr 2015 in Europa Zuflucht suchten, ist es wichtig zu untersuchen, wer diese Menschen sind. Bildung und Wertvorstellungen sind dabei wesentliche Aspekte. In diesem Beitrag werden das Humankapital, die Bildungslevels, beruflichen Qualifikationen, Einstellungen und Werte sowie die Gesundheit von Geflüchteten besprochen. Unsere Ergebnisse basieren vorwiegend auf DiPAS (Displaced Persons in Austria Survey), einer Befragung unter Geflüchteten, die 2015 größtenteils aus Syrien, Afghanistan und dem Irak nach Wien gekommen sind, und ReHIS (Refugee Health and Integration Survey), einer bundesweiten Gesundheitsbefragung unter Geflüchteten.

\section{2 „Displaced persons“ in der Forschung}

„Displaced persons“ sind laut der Internationalen Organisation für Migration (IOM) Personen, die wegen Bedrohung ihres Lebens, ihrer Freiheit oder ihrer Existenz ihre Heimatländer verlassen müssen. Dazu gehören Flüchtlinge (anerkannt laut der Genfer Flüchtlingskonvention von 1951), subsidiär Schutzberechtigte (mit zeitlich beschränktem humanitärem Schutz), Asylbewerber/innen (Menschen, die sich außerhalb der Staatsgrenzen ihres Ursprungslandes befinden und einen formellen Antrag auf 
internationalen Schutz gestellt haben, deren Verfahren noch läuft) sowie Binnenflüchtlinge (die innerhalb ihres Heimatlandes auf der Flucht sind).

Sowohl die Bedürfnisse von Geflüchteten als auch die Herausforderungen für die Aufnahmegesellschaften wurden trans- und interdisziplinär erforscht (AIYAR et al. 2016; Brun 2005; Keller et al. 2003; Robjant, Hassan \& Katona 2009; Thomas \& Thomas 2004; TURNER et al. 2003). Folgende Forschungsfelder stehen dabei im Vordergrund: besonders vulnerable Personengruppen wie Frauen und Kinder, Unterschiede in der kulturellen Identität, Inklusion und die gesellschaftliche Teilhabe von Geflüchteten im Aufnahmeland, Politikgestaltung und Aufnahmebedingungen sowie Rückkehrabsichten und zirkuläre Migration (Brun 2005; Derluyn \& Broekaert 2008; Doyle \& O‘Toole 2013; Fargues 2015; Halilovich 2013; Huemer, Karnik \& Steiner 2009; OECD 2016; Pię̧TKa-NykaZa 2015; Rosenberger \& König 2012; Ruiz, Siegel \& Vargas-Silva 2015; UNHCR 2013a). Dabei werden unterschiedliche methodische Ansätze verfolgt (sowohl qualitativ als auch quantitativ), wobei typischerweise jene Methoden angewandt werden, die in der jeweils eigenen Disziplin üblich sind (Bцосн 2007). Für Studien zu schwer erreichbaren Gruppen wie ethnischen Minderheiten wurden auch qualitative Erhebungstechniken (etwa snowball sampling) für die quantitative Forschung eingesetzt (Faugier \& Sargeant 1997; Singh \& Clark 2013).

\section{Die DiPAS-Datenerhebung}

Der Schwerpunkt von DiPAS (Displaced Persons in Austria Survey) lag auf Geflüchteten mit syrischer, irakischer und afghanischer Staatsbürgerschaft. Bei der Befragung, die im November und Dezember 2015 durchgeführt wurde, standen die soziodemographischen Charakteristika jener Menschen, die im Jahr 2015 in Österreich Zuflucht suchten, im Vordergrund. Die Erhebung erfolgte in sieben von NGOs betreuten Wohneinrichtungen in und um Wien (vier große Notquartiere und drei kleinere Unterkünfte). In jedem Quartier sprachen die Interviewer/innen Einzelpersonen an. Daneben wurde in größeren Notquartieren auch „,indirektes Snowballing“ angewandt. Die Daten wurden in Form einer computergestützten Face-to-face-Befragung (computer-assisted personal interviews $=$ CAPIs) erhoben. Fast zwei Drittel der Interviews wurden hauptsächlich auf Arabisch geführt, 20\% auf Farsi/Dari und 11\% auf Englisch.

Der DiPAS-Fragebogen basiert auf internationalen Surveys, wie dem LFS (Labour Force Survey), WVS (World Value Survey), SHARE (Survey of Health, Ageing and Retirement in Europe) und GGS (Generations and Gender Survey) (Börsch-SuPAN \& Jürges 2005; Vikat et al. 2007; World Value Survey 2016). Der Fragebogen war in fünf Themenblöcke gegliedert: Demographie, Humankapital, Erwerbstätigkeit, Gesundheit sowie Einstellungen und Wertehaltungen (KoHLENBERGER et al. 2017).

Insgesamt wurden 528 Interviews durchgeführt. Eine Beschränkung auf im Jahr 2015 angekommene Geflüchtete führte zu einer endgültigen Stichprobengröße von 514 
Personen (38\% mit irakischer, 36\% mit syrischer, 16\% mit afghanischer und $10 \%$ mit anderen Staatsbürgerschaften).

Die Datensätze enthalten auch Informationen über die Ehepartner/innen und Kinder, sodass insgesamt 1.391 Menschen in der Umfrage erfasst wurden, von denen 972 zur Zeit der Interviews in Österreich und 419 im Ausland lebten. Die Auskünfte zu Familienmitgliedern könnten einer gewissen Verzerrung unterliegen.

Setzt man die Familienmitglieder im Ausland in Relation zu den Angehörigen im Aufnahmeland, so ergibt sich ein Familienzusammenführungspotential von 0,38 (vgl. Abb. 3): Pro 100 Geflüchtete, die 2015 nach Österreich kamen, ist mit maximal 38 weiteren Personen zu rechnen, die für den Familienzuzug in Frage kommen. Dabei entfällt ein Großteil auf minderjährige Kinder (24) und Ehegattinnen (12). Volljährige Kinder sind formal nicht zur Familienzusammenführung berechtigt. Der Anteil der Ehegatten, die für eine Zusammenführung in Frage kommen, liegt in Österreich bei zwei Personen pro 100 Asylwerbenden (BUBER-ENNSER et al. 2016).

\section{Ab. 3: Potentieller Familiennachzug}

100 Asylwerber_innen, davon 30 minderjährige Kinder

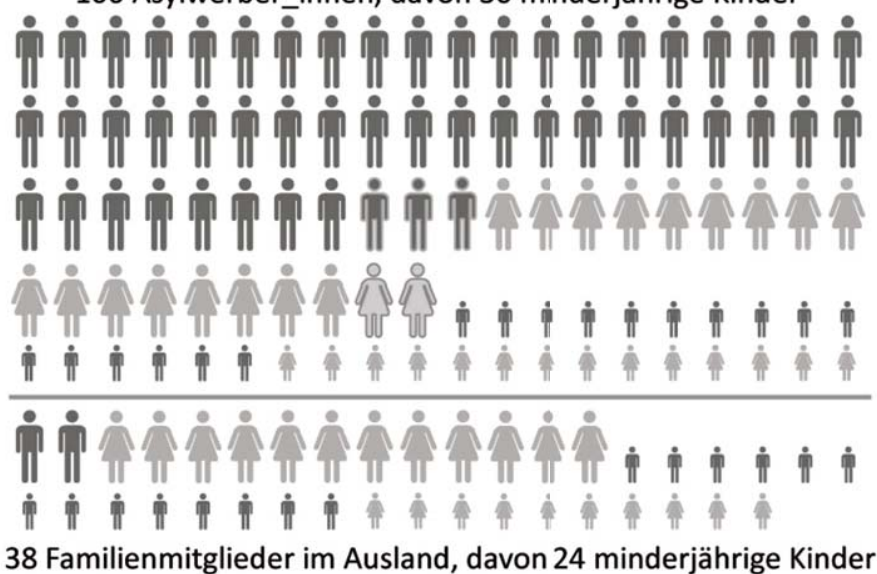

Quelle: DiPAS; BuBER-ENNSER et al. (2016).

\section{Das Bildungsniveau der Geflüchteten}

Auch wenn die These, dass Migrant/inn/en im Vergleich zur Gesamtbevölkerung ihres Herkunftslandes meist besser ausgebildet sind, nicht in allen Fällen zutrifft, bestätigt sie sich in Studien zu den Einwanderungsströmen in EU- und OECD-Länder (GrogGer \& Hanson 2011). Obwohl sich Flüchtlinge und Asylsuchende von Wirt- 
schaftsflüchtlingen unterscheiden, ist trotzdem zu erwarten, dass Menschen, die mehrere Grenzen überwinden, indem sie Schlepperorganisationen für ihre Flucht bezahlen, in ihren Heimatländern zur Mittelschicht gehörten und somit Zugang zu Bildung hatten. Die Ärmsten und daher vermutlich die am wenigsten Gebildeten könnten sich die Reise meist nicht leisten und sind stattdessen innerhalb ihres Heimatlandes oder in ein Nachbarland geflüchtet. Darüber hinaus könnten weniger Gebildete eher geneigt sein, zum Militär zu gehen als jene mit besserer Bildung, die auch Zugang zu Alternativen haben, einschließlich der Wehrdienstverweigerung, die in Syrien seit Beginn des Bürgerkriegs sehr häufig war.

Im Folgenden werden zuerst die Bildungsabschlüsse der Geflüchteten in der DiPAS-Stichprobe analysiert (Befragte, einschließlich Ehepartner/innen und erwachsene Kinder). Außerdem wird der Bildungsgrad der Personen in DiPAS mit dem im jeweiligen Herkunftsland (Syrien, Irak und Afghanistan) und in Österreich verglichen. Die Analyse bezieht die Qualität der Bildung nicht mit ein, sondern konzentriert sich auf quantitative Angaben. Allerdings zeigt eine Analyse des AMS (Arbeitsmarktservice Österreich) (2016), dass die Kompetenz- und Qualifikationsniveaus der Geflüchteten weitgehend mit dem Grad ihrer Schul- und/oder beruflichen Ausbildung übereinstimmen. Dies legt nahe, dass die in einigen Herkunftsländern erworbenen Fach-Qualifikationen als Resultat von Bildung, Ausbildung und einschlägiger Arbeitserfahrung - zumindest in den vom AMS getesteten Berufen - durchaus auf einem für den österreichischen Arbeitsmarkt adäquaten Niveau liegen.

In der DiPAS-Erhebung haben relativ wenige Personen keinerlei schulische Bildung genossen oder nur wenige Jahre eine Grundschule besucht: Der Anteil liegt bei etwa $15 \%$ und ist weitaus niedriger unter Geflüchteten aus Syrien (7\%) und dem Irak (9\%) als bei Menschen aus Afghanistan (25\%) (vgl. Abb. 4). Der überwiegende Anteil hat zumindest eine Pflichtschulbildung abgeschlossen (70\% der Befragten, Ehepartner/innen und erwachsenen Kinder). Die Unterschiede zwischen Männern und Frauen waren gering.

Bei den höchsten erzielten Bildungsabschlüssen ${ }^{1}$ zeigt sich Folgendes: $47 \%$ verfügen zumindest über eine höhere Sekundarbildung und 26\% über höhere Bildung (postsekundäre Bildung, also tertiäre Bildung wie Bachelorabschlüsse und kürzere postsekundäre Abschlüsse wie BHS-Abschlüsse) (vgl. Abb. 4). Der Anteil Höhergebildeter liegt bei den Personen aus dem Irak sogar bei 31\%, bei jenen aus Syrien bei 27\%. ${ }^{2}$

\footnotetext{
$1 \quad$ Bei der Erfassung des Bildungsgrads in DiPAS wurden keine Zeugnisse verlangt, weil dies den Interviewfluss behindert oder unterbrochen hätte. Da die Befragten erst kurz vor dem Interview in Österreich angekommen waren, hatten die meisten wohl noch keine Zeit für eine offizielle Übersetzung und Akkreditierung ihrer Zeugnisse durch österreichische Behörden.

2 Unterscheidet man weiter nach Bachelor-/Kollegabschluss und Master, so besitzt die überwiegende Mehrheit der Befragten mit Postsekundarbildung einen Bachelor-/Kollegabschluss (87\%). Einige der männlichen Befragten aus Syrien erwähnten im Interview, sie hätten ihr Studium absichtlich nicht abgeschlossen, um der Rekrutierung in die syrische Armee zu entgehen. Gleichzeitig äußerten junge Befragte den Wunsch, ihr Studium in Österreich abzuschließen (BUBER-ENNSER et al. 2016).
} 
Unterschiede im Bildungsgrad zwischen den Nationalitäten sind deutlich ausgeprägt: Während 53\% der syrischen und 46\% der irakischen Befragten zumindest eine höhere Sekundarbildung aufweisen, liegt dieser Anteil bei den Afghan/inn/en unter 30\%.

Abb. 4: Bildungsabschlüsse von Personen im Alter von 20 bis 59 Jahren in DiPAS

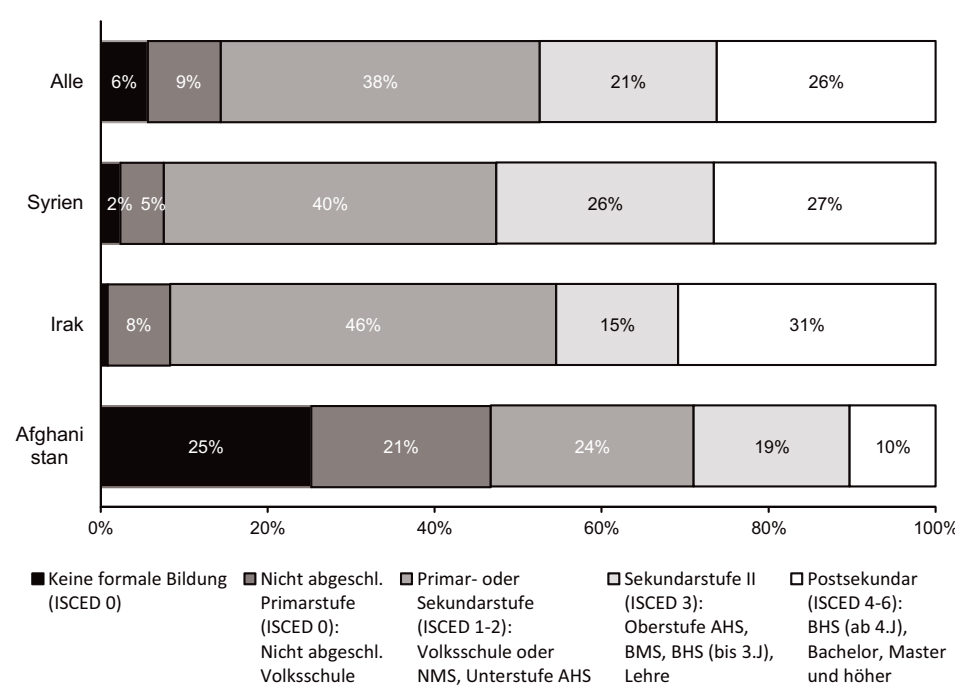

Quelle: DiPAS; BuBER-ENNSER et al. (2016).

Während die Bildungsergebnisse aus DiPAS in gewisser Weise eine Momentaufnahme aus dem Herbst 2015 darstellen, gehen sie doch über rein anekdotische Evidenz hinaus: Sämtliche 2016 oder 2017 in Österreich veröffentlichten Berichte lassen auf ein sehr ähnliches Bildungsprofil unter Flüchtlingen aus Syrien, dem Irak und Afghanistan schließen (vgl. Tabelle 1). Tatsächlich sind die erhobenen Prozentsätze in der Bildungsverteilung teilweise ident mit jenen aus DiPAS. Die Unterscheidung nach den drei größten Nationalitäten (Syrien, Irak, Afghanistan) führt zu sehr ähnlichen Ergebnissen. Auch jüngste Befunde aus Deutschland gelangen zu dem Schluss, dass Asylsuchende verschiedener Herkunft sehr heterogen sind und ein breites Spektrum zwischen Gering- und Hochqualifizierten abdecken: Geflüchtete, die in den letzten Jahren nach Europa gekommen sind, sind im Vergleich zur Bevölkerung ihrer Herkunftsländer besser gebildet, was sogar jene aus weniger entwickelten Ländern wie Afghanistan miteinschließt (WorBs, Bund \& BöHm 2016). Das Bildungsniveau syrischer Asylwerbender ist vergleichsweise hoch, weil dort bis zum Ausbruch des Bürgerkriegs im Jahr 2011 der Zugang zu Bildungseinrichtungen für alle gewährleistet war. Ein ähnlich hohes oder höheres Niveau wurde unter den Geflüchteten aus dem Iran und den Nachfolgestaaten der Sowjetunion beobachtet (BRÜCKER, ROTHER \& SCHUPP 2016). 
Tab. 1: Vergleich Bildungsabschlüsse von Geflüchteten in jüngsten Befragungen in Österreich und Deutschland

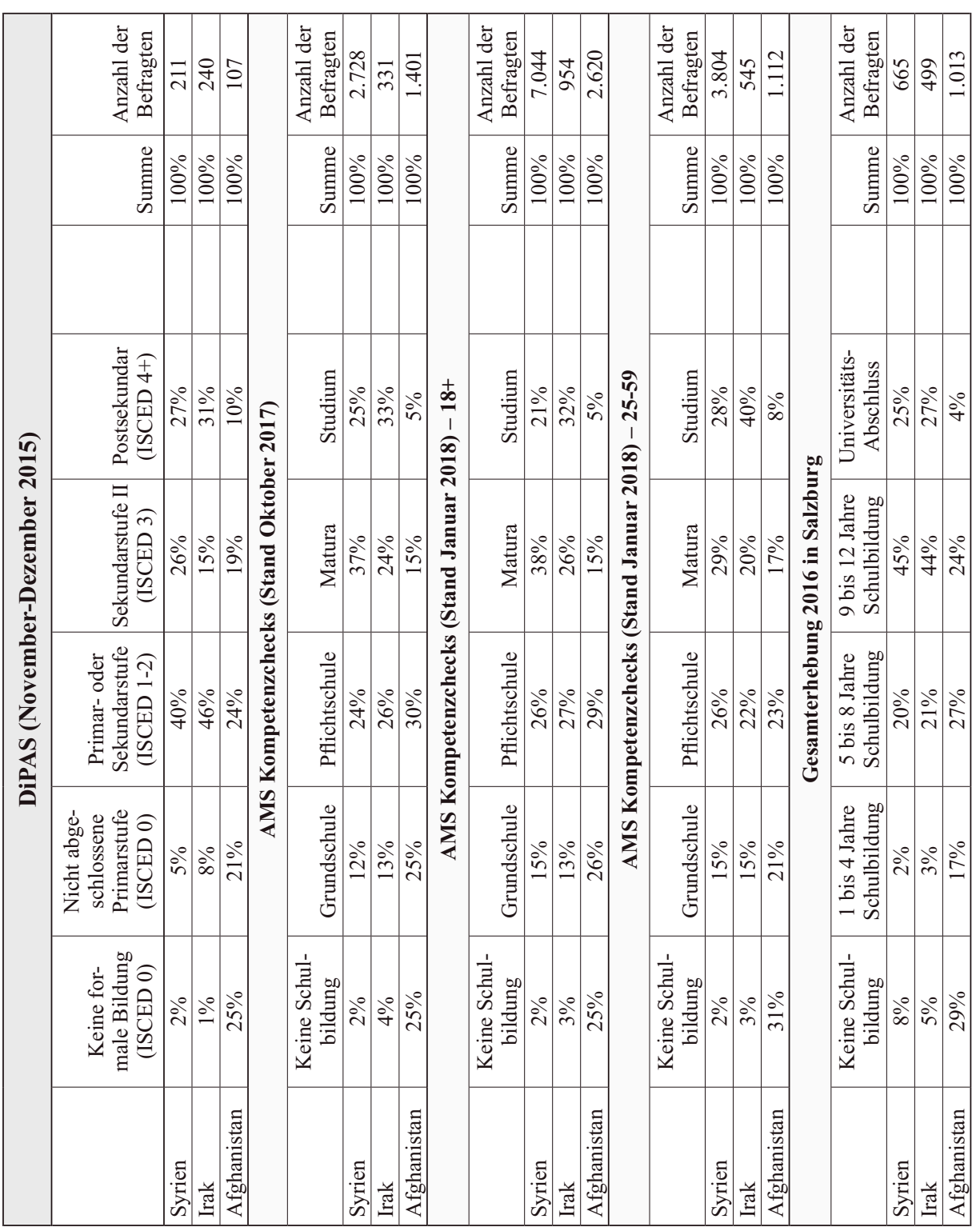




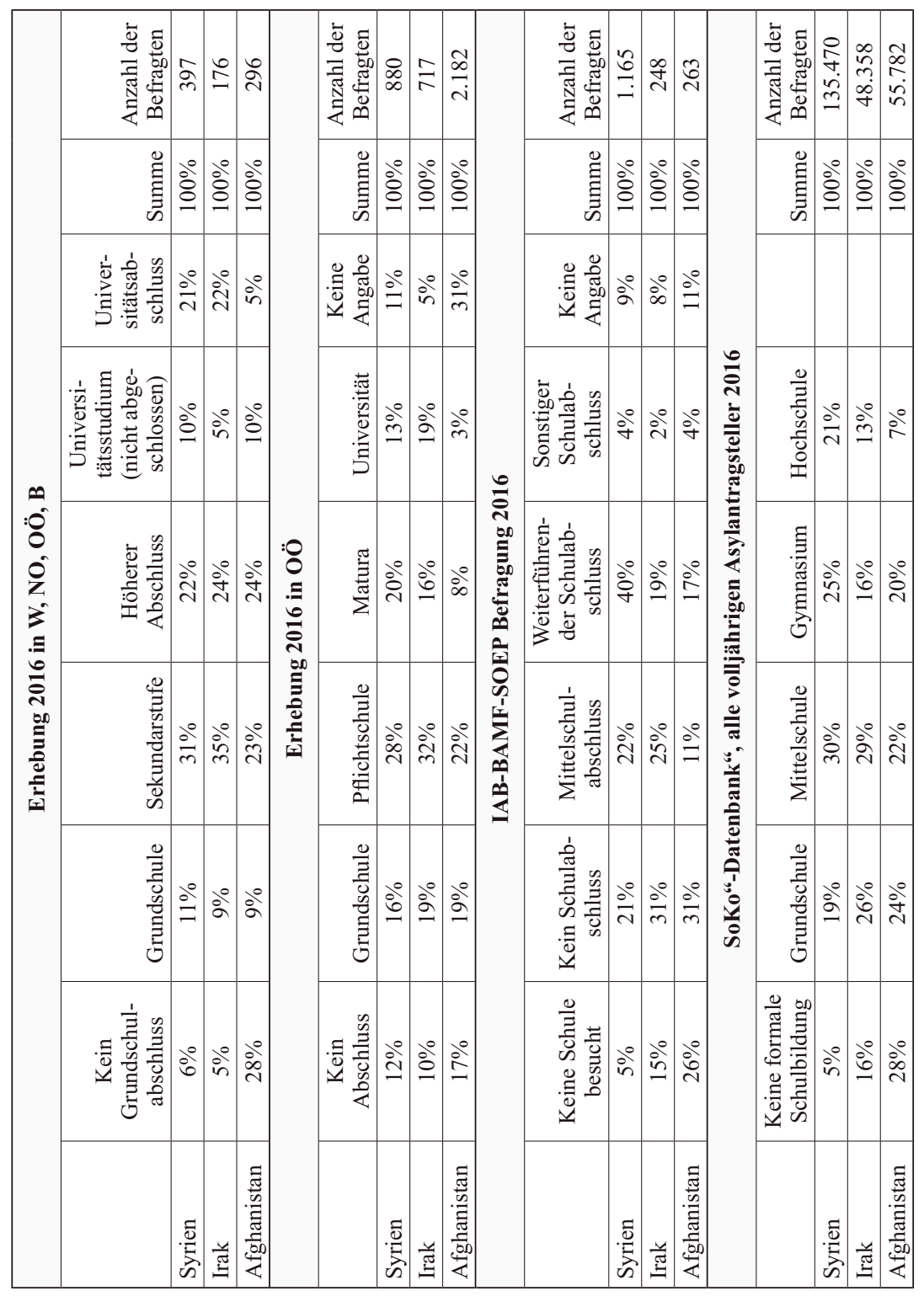

Quellen: AMS (2017); BrÜCKER et al. (2016); BubER-EnNSER et al. (2016); KoHlBACHER et al. (2017); Mitterndorfer (2017); NeSKe (2017); OÖ Landesregierung (2017); BubER-EnNSER \& KOHLENBERGER (2018). 
Abb. 5: Vergleich Bildungsabschlüsse von Personen in Österreich und in DiPAS

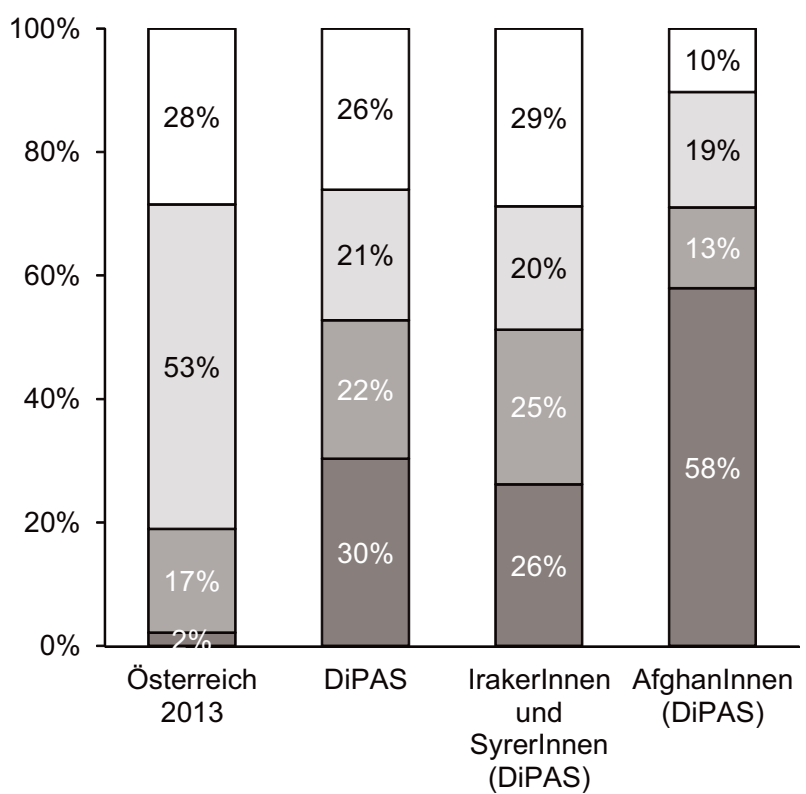

口Postsekundarstufe (ISCED 4-6):

BHS (ab 4.J), Bachelor, Master und höher

$\square$ Sekundarstufe II (ISCED 3):

Oberstufe AHS, BMS,

BHS (bis 3.J), Lehre

$\square$ Sekundarstufe I (ISCED 2):

NMS, Unterstufe AHS

$\square$ Primarstufe oder niedriger (ISCED 0-1):

keine formale Bildung,

nicht abgeschl. sowie

abgeschl. Volksschule

Quellen: Registerdaten für 2013, StATISTIK Austria und DiPAS; Buber-EnNSER et al. (2016).

Ein Vergleich mit der österreichischen Bevölkerung zeigt, dass der Prozentsatz hoch gebildeter Inländer/innen vergleichbar mit dem Anteil in DiPAS ist, vor allem unter syrischen und irakischen Staatsangehörigen (29\% verglichen mit $28 \%$ in der österreichischen Bevölkerung) (vgl. Abb. 5). Aber der Anteil der Geflüchteten mit abgeschlossener höherer Sekundarbildung (21\%) ist nur halb so hoch wie unter Österreicher/ inne/n (53\%), während der Anteil jener, die höchstens eine abgeschlossene Primarbildung aufweisen, in DiPAS weitaus höher ist als in der österreichischen Bevölkerung (vgl. Abb. 5).

Geschlechtsspezifische Bildungsunterschiede sind relativ gering. Ein deutlicher Unterschied zeigt sich bei Personen ohne Schulbildung, bei denen Frauen mit 10\% einen höheren Anteil stellen als Männer (4\%).

Aufgrund des selektiven Effekts von Migration ist zu erwarten, dass Geflüchtete besser gebildet sind als die Gesamtbevölkerung in ihrem Heimatland. Der Vergleich dieser beiden Gruppen ist aufgrund der mangelhaften Datenlage nicht leicht. Dennoch zeigt der hohe Anteil von Geflüchteten mit postsekundärer Bildung, dass sie sich deutlich von der Gesamtbevölkerung unterscheiden. Die syrischen und afghanischen DiPAS- 
Befragten sind weitaus höher gebildet als die Gesamtbevölkerung ihrer Heimatländer (vgl. Abb. 6). ${ }^{3}$

Obwohl afghanische DiPAS-Teilnehmer/innen ein weitaus niedrigeres Bildungsniveau aufweisen als syrische und irakische Befragte, sind sie dennoch viel besser gebildet als (gleichaltrige) Erwachsene in Afghanistan. Das generell niedrigere Bildungsniveau der Afghan/inn/en erklärt sich aus den schwierigen Rahmenbedingungen, denen das afghanische Bildungssystem jahrzehntelang unterworfen war - einerseits wegen des Kriegszustands nach der sowjetischen Invasion 1979, aber auch weil mit der Machtergreifung der Taliban Ende der 1990er-Jahre ein besonders bildungsfeindliches Regime im Land installiert wurde.

\section{Abb. 6: Bildungsabschlüsse in den Heimatländern und in DiPAS}

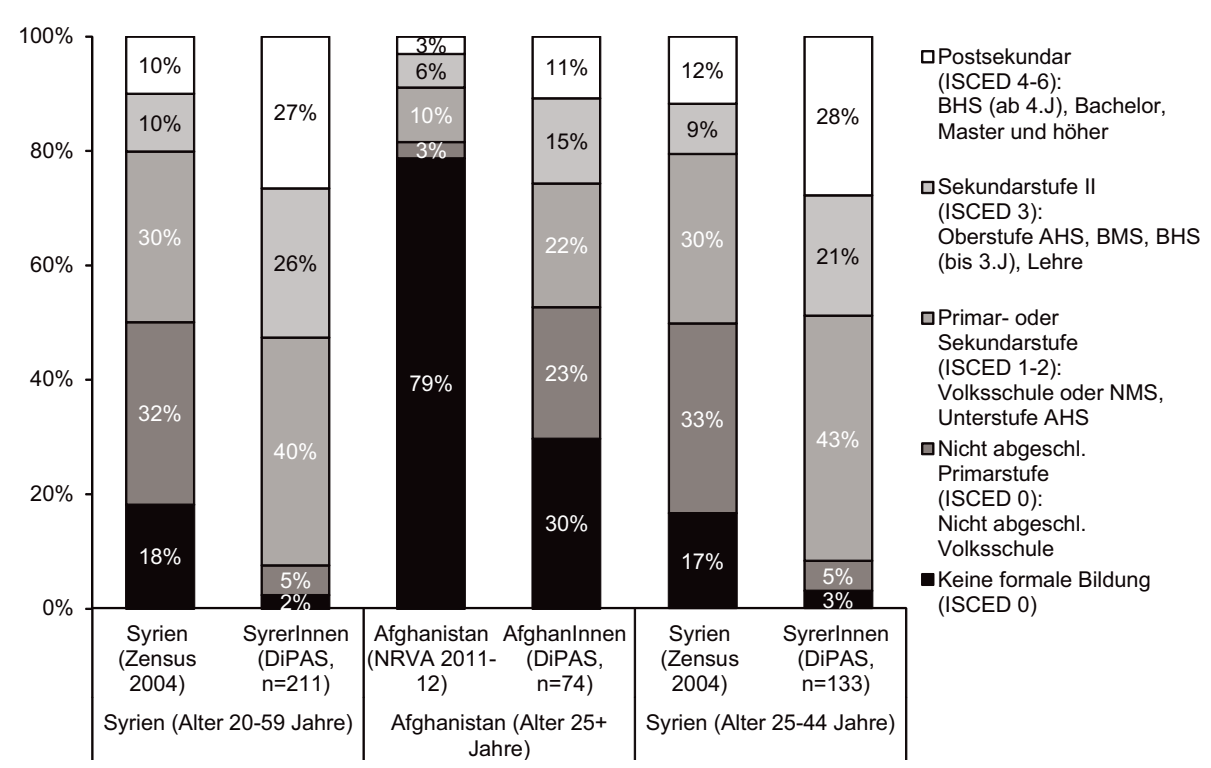

Quellen: DiPAS, Buber-Ennser et al. (2016), Central Bureau of Statistics (2004) für Syrien, Central Statistics Organisation (2014) für Afghanistan.

Anmerkung: Für die irakische Bevölkerung ist keine repräsentative Umfrage aus der näheren Vergangenheit verfügbar. Das Altersspektrum spiegelt die Datenverfügbarkeit wider.

Für die Syrer/innen beruht dieser Vergleich auf den letzten verlässlichen Zensusdaten des Jahres 2004. Da die Menschen im Heimatland durchschnittlich älter sind als die Personen in DiPAS, wurde der Vergleich für Syrien auf die Altersgruppe 25-44 beschränkt, wodurch wir aber ähnliche Ergebnisse erhalten (vgl. Abb. 6, rechter Teil). 


\section{Werte und Einstellungen}

Ein weiterer wichtiger Bereich in der Aufnahme und Integration von Geflüchteten betrifft ihre Werte und Einstellungen. Eine häufig geäußerte Sorge ist, dass Flüchtlinge, vor allem Personen mit muslimischem Glauben, westliche Werte komplett ablehnen und sich damit der Integration im Aufnahmeland verweigern könnten (BAWER 2007; FETZER \& SOPER 2005). Gerade die religiöse Identität von Geflüchteten wurde bereits zu Beginn des Fluchtherbstes 2015 medial und politisch heftig diskutiert (CARRERA et al. 2015; CULIK 2015). Das ist wenig überraschend, da in den meisten der europäischen Aufnahmeländer negative, zumeist diskriminierende und häufig offen feindselige Einstellungen gegenüber Menschen muslimischen Glaubens vorherrschen. Gleichzeitig gibt es weitreichende Hinweise auf eine „Akkulturation” von migrantischen Gruppen: Im Laufe der Zeit tendieren Migrant/inn/en dazu, ihre Einstellungen zur Religion und Geschlechtergerechtigkeit an jene des Aufnahmelands anzupassen, ohne zwangsläufig ihren religiösen Glauben aufzugeben (NORRIS \& IngLeHART 2012; RöDER \& MüHLAU 2014). Es ist jedoch unklar, ob das auch auf Menschen zutrifft, die nicht freiwillig auswandern, sondern aufgrund von Krieg und Verfolgung aus ihrem Herkunftsland flüchten mussten. Um ein umfassenderes Bild zu den bisher wenig untersuchten Wertvorstellungen von Geflüchteten aus dem Herbst 2015 zu erlangen (cf. KOHLBACHER et al. 2017), wurden DiPAS-Teilnehmende auch nach ihren Einstellungen zu Geschlechtergleichheit, Schwangerschaftsabbruch, weiblicher Erwerbstätigkeit, Religionsunterricht in der Schule und ihrer Religiosität befragt.

Bei der Frage nach der eigenen Religiosität war die Intensität des Glaubens auf einer Skala von 1 (überhaupt nicht religiös) bis 10 (sehr religiös) einzustufen. Viele Befragte empfanden es als schwierig, ihre Religiosität zu quantifizieren und ein groBer Teil der Befragten (40\%) wählte 5, den Mittelwert. ${ }^{4}$ In Übereinstimmung mit der Literatur stuften sich Frauen religiöser ein als Männer (vgl. Tab. 2). Der Anteil der sehr religiösen Personen sinkt mit steigender Bildung. Mehr Flüchtlinge gaben an nicht religiös zu sein (20\%) als sehr religiös (11\%) (vgl. Tab. 2). Diese Verteilung entspricht grob jener der österreichischen Wohnbevölkerung: In der Altersgruppe von 18 bis 45 Jahren bezeichnet sich jede/r zehnte Österreicher/in als sehr religiös. Im Gegensatz dazu gaben $23 \%$ geringe Religiosität an, während zwei von drei Personen ihre Religiosität als mittel einschätzten (ebenfalls Tab. 2). Trotz der leicht unterschiedlichen Skalen ergibt sich eine sehr ähnliche Verteilung der religiösen Levels bei Geflüchteten und Österreicher/innen: Jede/r Zehnte stuft sich selbst als sehr religiös ein, während 20\% eine niedrige Religiosität angeben und die große Mehrheit (69\%) ihre Religiosität als mittel beurteilt.

\footnotetext{
Auch scheint eine gewisse Interviewer/innenverzerrung vorhanden zu sein, da Befragte, die das Interview in ihrer Muttersprache absolvierten, ihre Religiosität im Schnitt als stärker einstuften als jene, die auf Englisch befragt wurden.
} 
Tab. 2: Einschätzung der eigenen Religiosität unter den befragten Geflüchteten und der österreichischen Bevölkerung, nach Geschlecht.

\begin{tabular}{|l|c|r|r|r|}
\hline & Religiosität & Männer & Frauen & Gesamt \\
\hline Geflüchtete & Wenig (1-2) & $24 \%$ & $4 \%$ & $20 \%$ \\
\hline & Mittel (3-8) & $67 \%$ & $78 \%$ & $69 \%$ \\
\hline & Sehr (9-10) & $9 \%$ & $18 \%$ & $11 \%$ \\
\hline Österreicher/innen & Gesamt & $100 \%$ & $100 \%$ & $100 \%$ \\
\hline & Wenig (0-2) & $27 \%$ & $20 \%$ & $23 \%$ \\
\hline & Mittel (3-8) & $66 \%$ & $66 \%$ & $66 \%$ \\
\hline & Sehr (9-10) & $7 \%$ & $14 \%$ & $10 \%$ \\
\hline & Gesamt & $100 \%$ & $100 \%$ & $100 \%$ \\
\hline
\end{tabular}

Quellen: DiPAS; Generations and Gender Survey (GGS) 2008/9; Buber-Ennser et al. (2018).

Die eingangs erwähnte „Akkulturation“ bezüglich der Geschlechterrollen hat einen starken Effekt auf die Arbeitsmarktintegration geflüchteter Frauen (BuRDA, HAMERMESH \& WeIl 2012; Hall \& Zoega 2014), da Migrant/inn/en aus Ländern mit weniger egalitären Geschlechterrollen eine niedrigere Frauenerwerbsquote und höhere Fertilitätsraten aufweisen (Blau et al. 2013). Diese Effekte können zu einem signifikanten interethnischen, geschlechterspezifischen Lohngefälle führen (ANTECOL 2001). Für die Teilnahme von geflüchteten Frauen am Arbeitsmarkt des Aufnahmelandes ist die Einstellung zur weiblichen Erwerbstätigkeit unter Geflüchteten zentral, welche in DiPAS u.a. durch folgende Aussage erhoben wurde, welcher die Befragten entweder zustimmen oder sie ablehnen sollten: „Wenn Arbeit knapp ist, sollten Männer mehr Anspruch auf einen Job haben als Frauen“. Die Unterschiede in den Antworten von Männern und Frauen waren gering, da beide Geschlechter zu knapp 50\% der Aussage zustimmten (vgl. Abb. 7, linke Grafik). Frauen wählten häufiger sowohl ,stimme zu“ als auch „lehne ab“ und vermieden damit die neutrale Option, wobei die Zahl der weiblichen Befragten in DiPAS niedrig ist. Der Vergleich mit dem irakischen World Value Survey (WVS) zeigt, dass die Männer in der DiPAS-Stichprobe weitaus weniger traditionell eingestellt sind: Während unter der irakischen Gesamtbevölkerung 70\% der Männer der Aussage zustimmen, sind es in DiPAS nur 48\% der männlichen Befragten. 
Abb. 7: „Wenn Arbeit knapp ist, sollten Männer mehr Anspruch auf einen Job haben als Frauen."

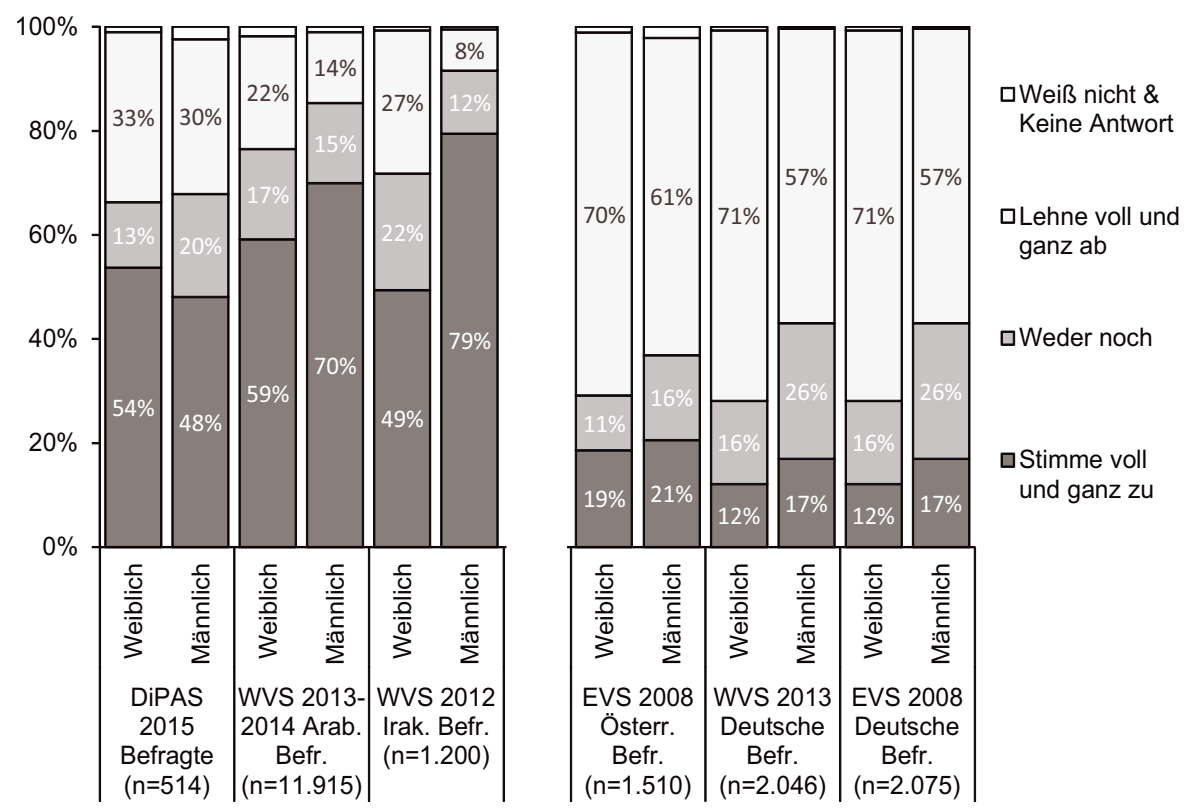

Quellen: WVS; BubER-EnNSER et al. (2016); DiPAS

Anmerkung: „Arabische Befragte“ schließt Menschen aus Algerien, Bahrain, Ägypten, Jordanien, Kuwait, Libanon, Libyen, Tunesien und Jemen ein.

In Österreich und Deutschland lag die Ablehnung der Aussage „Wenn Arbeit knapp ist, sollten Männer mehr Rechte auf einen Job haben als Frauen" bereits im Jahr 2008 bei über 60\% (für beide Geschlechter) (vgl. Abb. 7, rechte Grafik). Die DiPASStichprobe zeigt jedoch, dass befragte Männer die Aussage häufiger ablehnen als arabische oder irakische Männer in der WVS-Stichprobe. Dies könnte bedeuten, dass männliche Erwachsene, die Frauenrechte unterstützen, eher dazu tendierten ihr Heimatland zu verlassen. Diese Interpretation wird auch durch das hohe Bildungsniveau der DiPASBefragten gestützt, da eine weniger traditionelle Einstellung generell mit einem höheren Bildungsgrad assoziiert ist.

\section{Gesundheit}

Die ,healthy immigrant theory“ (Domnich et al. 2012; Marmot, Adelstein \& Bulusu 1984) besagt, dass unter Migrant/innen ein „Selbstselektionseffekt“ besteht, sodass diese in der Regel bei besserer Gesundheit sind als ihre Landsleute im Heimat- 
land einerseits sowie die Einheimischen des Ziellandes andererseits. Umgekehrt ist es aber wenig wahrscheinlich, dass Bürgerkriegsopfer und Asylsuchende, die vor Gewalt und Verfolgung fliehen, auch in dieses Muster fallen, da sich ihre Migrationsgründe stark von jenen regulärer Migrant/inn/en unterscheiden (KoHLs 2011). Dennoch scheinen die DiPAS-Befragten sehr gesund zu sein: Die meisten bezeichneten ihren Gesundheitszustand als ,gut” oder „sehr gut“ (85\%), gaben keine chronischen Krankheiten (84\%) oder Arbeitsunfähigkeit aufgrund von gesundheitlichen Problemen (83\%) an.

Generell schätzen Frauen ihren Gesundheitszustand schlechter ein als Männer: Nur 69\% der Frauen gegenüber 89\% der Männer bezeichneten ihre Gesundheit als "gut" oder "sehr gut". Dieses Geschlechtergefälle kann nicht nur unter Migrant/inn/en beobachtet werden, sondern tritt auch in autochthonen Populationen auf (BENYAMINI et al. 2003). Bei der Interpretation dieses Geschlechtergefälles ist zu bedenken, dass weibliche Befragte im Durchschnitt drei Jahre älter waren als männliche (das Durchschnittsalter beträgt 33,3 Jahre für Frauen und 30,5 für Männer) und dass die kleine Stichprobe derartige Auswertungen beeinträchtigen könnte. Ein Vergleich mit Österreicher/innen (BMG 2015) zeigt Geschlechtsunterschiede: Geflüchtete Männer bezeichneten sich als gesünder und geflüchtete Frauen als weniger gesund als die generelle österreichische Bevölkerung (Männer: 89\% gegenüber 81\%; Frauen: 69\% gegenüber 77\%).

Eine weitere Erhebung, die Anfang 2018 durchgeführt wurde, verstärkt dieses Bild: Primärdaten aus dem Refugee Health and Integration Survey (ReHIS), einer sozialwissenschaftlichen Erhebung unter 515 hauptsächlich syrischen, irakischen und afghanischen Geflüchteten in Österreich, zeigt, dass Geflüchtete, insbesondere Frauen und Menschen aus Afghanistan, dazu tendieren, ihre Gesundheit schlechter einzuschätzen als die österreichische Bevölkerung (KoHLENBERGER et al. 2019). Obwohl sich Geflüchtete generell sehr zufrieden mit dem österreichischen Gesundheitssystem zeigen und nur vereinzelt formale Zugangsbarrieren genannt wurden, erwähnten zwei von zehn männlichen und vier von zehn weiblichen ReHIS-Befragten ungedeckte Gesundheitsbedürfnisse. Die am häufigsten genannten Barrieren des Zugangs zum Gesundheitssystem waren Terminkonflikte, lange Wartelisten, mangelndes Wissen über Ärzt/innen im unmittelbaren Umfeld und Sprachbarrieren. Gerade jene fachärztlichen Behandlungen, die von Geflüchteten sehr selten in Anspruch genommen wurden, sind mit einem Selbstbehalt verbunden, wie z.B. die Mehrheit der präventiven Zahnbehandlungen sowie Physiotherapie. Gleichzeitig gaben Geflüchtete Krankenhausbehandlungen wesentlich öfter an als Österreicher/inn/en, wobei ambulante Behandlungen häufiger waren als stationäre Aufenthalte.

\section{Diskussion}

Die präsentierten Ergebnisse sollen zu einer evidenzbasierten Diskussion über Geflüchtete beitragen und Antworten auf die Frage geben, wer 2015 nach Österreich 
flüchtete: Anstatt zu fragen „Wie viele sind gekommen?“, geht es mehr und mehr um die Frage „Wer ist gekommen?“.

Die hier vorgestellten Ergebnisse betreffen vorrangig die Bildung, das Wertesystem und die Gesundheit von Geflüchteten in Österreich. Speziell die syrischen und irakischen Befragten, die im Sommer und Herbst 2015 ankamen, weisen einen konsistent hohen Bildungsgrad auf, was die weit verbreitete öffentliche Annahme, Asylsuchende und Flüchtlinge seien ungebildet oder gar Analphabet/inn/en, widerlegt (BUBER-ENNSER \& KoHLENBERGer 2018). Der Anteil der Befragten mit keiner oder nur geringer formaler Bildung stellte sich als sehr niedrig heraus. Asylsuchende verschiedener Herkunft weisen unterschiedliche Charakteristika auf und sogar jene aus weniger entwickelten Ländern wie Afghanistan sind im Vergleich zur Bevölkerung ihrer Herkunftsländer positiv selektiert.

Bezüglich des Familienkontextes zeigt sich, dass die Mehrheit mit ihrer Kernfamilie geflüchtet ist, besonders in der syrischen Gruppe. Die größte Wahrscheinlichkeit für Familienzusammenführungen besteht für Mütter und minderjährige Kinder, die sich ihren bereits emigrierten Familienmitgliedern anschließen könnten: Nach unseren Schätzungen besteht das Potenzial für solche Familienzusammenführungen vor allem aus Kindern unter 18 Jahren (62\%) und Ehepartner/inne/n (38\%).

Die Ergebnisse zu Einstellungen und Werten der Geflüchteten, vor allem ihre Antworten zu Religiosität und der (beruflichen) Rolle von Frauen, können zur aktuellen Debatte über Einstellungen zu Religion und Geschlechtergerechtigkeit beitragen. Anders als gemeinhin wohl erwartet würde, lehnten sowohl weit mehr männliche als auch weibliche Befragte traditionelle Einstellungen ab als dies in ihren Herkunftsländern der Fall ist. Der Großteil stufte die eigene Religiosität in einem mittleren Bereich ein, zwei von zehn bezeichneten sich als nicht religiös, nur halb so viele als sehr religiös. In Übereinstimmung mit früheren Forschungsergebnissen tendierten weibliche Befragte dazu, sich als religiöser einzuschätzen als die Männer.

Der vorliegende Artikel ist eine Zusammenschau von Ergebnissen basierend auf DiPAS und ReHIS, zwei aktuellen Erhebungen unter Geflüchteten in Österreich. Sie zeigen, dass die empirische Forschung Antworten bietet auf die Frage, wer 2015 nach Österreich gekommen ist. Aktuell werden auch weitere quantitative Erhebungen analysiert, die auf Arbeitsmarkt und Integration in den Arbeitsmarkt sowie die Gesellschaft fokussieren. Neben diesen quantitativen Ansätzen gibt es zahlreiche qualitative Forschungsprojekte (Kaufmann 2016; Kohlbacher \& Schiocchet 2017), die die religiöse und kulturelle Identität, die Wertvorstellungen und das Integrationspotential geflüchteter Menschen aus dem Herbst 2015 untersuchen. 


\section{Bibliographie}

Aiyar S., Barkbu B., Batini N. et al. (2016), The refugee surge in Europe: Economic challenges. Washington DC, International Monetary Fund.

AMS (2016), Asylberechtigte auf Jobsuche. Kompetenzcheck-Ergebnisse und Integrationsmaßnahmen im Jahr 2016 [Persons granted asylum looking for a job. Competence check results and integration measures in the year 2016]. Vienna, Public Employment Service Austria (AMS).

AMS (2017), Arbeitsmarktintegration geflüchteter Menschen: Bilanz und Ausblick. Pressekonferenz.

Antecol H. (2001), Why is there interethnic variation in the gender wage gap?: The role of cultural factors. The Journal of Human Resources, 36 (1), pp. 119-143.

BAMF (2016a), Aktuelle Zahlen zu Asyl. Ausgabe: März 2016 [Recent numbers on asylum: March 2016]. Nürnberg, Bundesamt für Migration und Flüchtlinge [Federal Office for Migration and Refugees].

BAMF (2016b), Asylgeschäftsstatistik für den Monat Dezember 2015 [Asylum statistics for December 2015]. Nürnberg, Bundesamt für Migration und Flüchtlinge [Federal Office for Migration and Refugees].

BAwER B. (2007), While Europe slept: How radical Islam is destroying the West from within. New York, Broadway Books.

Benyamini Y., Blumstein T., Lusky A., et al. (2003), Gender differences in self-rated healthmortality association: Is it poor self-rated health that predicts mortality or excellent selfrated health that predicts survival? The Gerontologist, 43, pp. 396-405.

Blau F.D., Kahn L.M., Yung-Hsu Liu A., et al. (2013), The transmission of women's fertility, human capital, and work orientation across immigrant generations. Journal of Population Economics, 26 (2), pp. 405-435.

BLOCH A. (2007), Methodological challenges for national and multi-sited comparative survey research. Journal of Refugee Studies, 20 (2), pp. 230-247.

BMG (2015), Österreichische Gesundheitsbefragung 2014. Hauptergebnisse des Austrian Health Interview Survey (ATHIS) und methodische Dokumentation [Austrian Health Survey 2014. Main results of the Austrian Health Interview Survey (ATHIS) and methodological documentation]. Vienna, Austrian Federal Ministry of Health.

BMI (2016), Asylstatistik 2015 [Asylum statistics 2015]. Vienna, Austrian Federal Ministry of the Interior.

BMI (2018), Asylstatistik 2017 [Asylum statistics 2017]. Vienna, Austrian Federal Ministry of the Interior.

Börsch-Supan A. \& Jürges H. (eds.) (2005), The Survey of Health, Ageing and Retirement in Europe - Methodology. Mannheim, Mannheim Research Institute for the Economics of Aging.

BRÜCKER H., Rother N. \& SCHUPP J. (eds.) (2016), IAB-BAMF-SOEP-Befragung von Geflüchteten: Überblick und erste Ergebnisse [IAB-BAMF-SOEP-Survey on refugees: Overview and first results]. Research Report 14/2016, Nürnberg, Institute of Employment Research (IAB).

BRUN C. (2005), Women in the local/global fields of war and displacement. Gender, Development and Technology, 9 (1), pp. 57-80. 
Buber-Ennser I., Goujon A., Kohlenberger J. et al. (2018), Multi-layered roles of religion among refugees arriving in Austria around 2015. Religions, 9 (5), p. 154.

Buber-Ennser I. \& Kohlenberger J. (2018), These: „Die AsylwerberInnen der letzten Jahre sind meist AnalphabetInnen“ [Thesis: „Persons seeking asylum during the last years are mostly illiterate"]. In: Haller M. (ed.), Migration \& Integration. Fakten oder Mythen? Siebzehn Schlagwörter auf dem Prüfstand [Migration \& integration. Facts or myths? Seventeen buzzwords put to the test]. Vienna, Commission for Migration of the Austrian Academy of Sciences, (pp. 123-135).

Buber-Ennser I., Kohlenberger J., Rengs B. et al. (2016), Human capital, values, and attitudes of persons seeking refuge in Austria in 2015. PLoS ONE, 11 (9), pp. e0163481.

Burda M., Hamermesh D.S. \& Weil P. (2012), Total work and gender: Facts and possible explanations. Journal of Population Economics, 26 (1), pp. 239-261.

Carrera S., Blockmans S., Gros D., et al. (2015), The EU's response to the refugee crisis: Taking stock and setting policy priorities CEPS Essay No. $20 / 16$ December 2015. Brussels, Centre for European Policy Studies.

Central Bureau of Statistics (2004), Census 2004. Damascus: CBS. Retrieved 24 October 2018, from <http://www.cbssyr.sy/General\%20census/census $\% 202004 /$ General $\% 20$ census $\% 20$ tab2.htmhttp://www.cbssyr.sy/General $\% 20$ census/census $\% 202004 /$ General $\% 20$ census\%20tab2.htm>

Central Statistics Organisation (2014), National risk and vulnerability assessment 2011-2012. Afghanistan Living Conditions Survey. Kabul, CSO.

Culik J. (2015), Anti-immigrant walls and racist tweets: The refugee crisis in central Europe. The Conversation, 24 June 2015, retrieved 2 December 2018, from $<$ http://theconversation. com/anti-immigrant-walls-and-racist-tweets-the-refugee-crisis-in-central-europe-43665>

Derluyn I. \& Broekaert E. (2008), Unaccompanied refugee children and adolescents: The glaring contrast between a legal and a psychological perspective. International Journal of Law and Psychiatry, 31 (4), pp. 319-330.

Domnich A., Panatoo D., Gasparini R., et al. (2012), The "healthy immigrant" effect: Does it exist in Europe today? Italian Journal of Public Health, 9 (3), pp. e7532-7531-e7532-7537.

Doyle L. \& O‘Toole G. (2013), A lot to learn: Refugees, asylum seekers and post-16 learning. London, British Research Council.

EJPD (2016), Asylstatistik 2015 [Asylum statistics 2015]. Bern-Wabern, Eidgenössisches Justizund Polizeidepartement EJPD [Federal Department of Justice and Police].

EUROSTAT (2016), Your key to European statistics. Retrieved 6 February 2018, from <http:// ec.europa.eu/eurostat/web/asylum-and-managed-migration/data/database>

FARGues P. (2015), The year we mistook refugees for invaders. Florence, Migration Policy Center.

FASSMANN H. \& STACHER I. (eds.) (2003), Österreichischer Migrations- und Integrationsbericht. Demographische Entwicklungen - sozioökonomische Strukturen - rechtliche Rahmenbedingungen [Austrian migration and integration report. Demographic developments socioeconomic situation - legal framework]. Klagenfurt/Celovec, Drava.

Faugier J. \& Sargeant M. (1997), Sampling hard to reach populations. Journal of Advanced Nursing, 26 (4), pp. 790-797.

Fetzer J. S. \& Soper C. (2005), Muslims and the state in Britain, France, and Germany. Cambridge, Cambridge University Press. 
Franz B. (2005), Uprooted and unwanted: Bosnian refugees in Austria and the United States. College Station, Texas, A\&M University Press.

Grogger J. \& Hanson G. H. (2011), Income maximization and the selection and sorting of international migrants. Journal of Development Economics, 95, pp. 42-57.

Halilovich H. (2013), Bosnian Austrians: Accidental migrants in trans-local and cyber spaces. Journal of Refugee Studies, 26 (4), pp. 524-540.

Hall A. \& Zoega G. (2014), Values and labor force participation in the Nordic countries. Economics, 8 (41), pp. 1-43.

Huemer J., Karnik N. \& Steiner H. (2009), Unaccompanied refugee children. The Lancet, 373 (9664), pp. 612-614.

Kaufmann K. (2016), Wie nutzen Flüchtlinge ihre Smartphones auf der Reise nach Europa? Ergebnisse einer qualitativen Interview-Studie mit syrischen Schutzsuchenden in Österreich [How do refugees use their smart-phones on their way to Europe? Results of qualitative interviews with Syrian refuge seeking persons in Austria]. SWS-Rundschau, 56 (3), pp. 319-342.

Keller A.S., Rosenfeld B., Trinh-Shevrin C., et al. (2003), Mental health of detained asylum seekers. The Lancet, 362 (9397), pp. 1721-1723.

Kohlbacher J., Rasuly-Paleczek G., Hackl A., et al. (2017), Wertehaltungen und Erwartungen von Flüchtlingen in Österreich. Endbericht [Attitudes and expectations of refugees in Austria. Final report]. Vienna, Federal Ministry for Europe, Integration and Foreign Affairs (BMEIA).

Kohlbacher J. \& Schiocchet L. (2017), From destination to integration: Afghan, Syrian and Iraqi refugees in Vienna. Vienna, Austrian Academy of Sciences Press.

Kohlenberger J., Buber-Ennser I., Rengs B., et al. (2017), A social survey on asylum seekers in and around Vienna in fall 2015: Methodological approach and field observations. Refugee Survey Quarterly, 36 (4), pp. 90-109.

Kohlenberger J., Buber-Ennser I., Rengs B., Leitner S., \& Landesmann M. (2019), Barriers to health care access and service utilization of refugees in Austria: Evidence from a crosssectional survey. Health Policy, online first. $<$ doi:10.1016/j.healthpol.2019.01.014>

Kohls M. (2011), Morbidität und Mortalität von Migranten in Deutschland [Morbidity and Mortality of migrants in Germany]. Nürnberg, Bundesamt für Migration und Flüchtlinge [Federal Office for Migration and Refugees].

Marik-LebeK S. \& Wisbauer A. (2017), Flüchtlingsmigration im Spiegel der Bevölkerungsstatistik [Refugee migration mirrowed in vital statistics]. Statistische Nachrichten, 2017 (4), pp. 268-275.

Marmot M.G., Adelstein A.M. \& Bulusu L. (1984), Lessons from the study of immigrant mortality. The Lancet, 323 (8392), pp. 1455-1457.

Migrationsverket (2016), Asylum applications. Stockholm, Migrationsverket [Swedish Migration Agency].

MitTERNDORFER P. (2017), Qualifikations-Screening von Asylwerbenden in der Grundversorgung des Landes Salzburg 2016 [Qualification screening of asylum seekers receiving basic supply in the Federal state of Salzburg 2016]. Salzburg, Landesamtsdirektion.

NeSKe M. (2017), Sozialstruktur, Qualifikationsniveau und Berufstätigkeit. Volljährige Asylerstantragsteller in Deutschland im Jahr 2016 [Social structure, qualification and employment. Adult asylum seekers in Germany in 2016]. Nürnberg, Bundesamt für Migration und Flüchtlinge [Federal Office for Migration and Refugees]. 
NorRis P. \& IngLeharT R. (2012), Muslim integration into Western cultures: Between origins and destinations. Political Studies, 60 (2), pp. 228-251.

OÖ LANDESREgIERUNG (2017), Qualifikation Asylwerber/innen [Qualification of asylum seekers]. Linz, Amt der OÖ Landesregierung [Office of the provincial governement of Upper Austria].

OECD (2016), Making integration work: Refugees and others in need of protection. Paris, OECD Publishing.

PięTKa-Nykaza E. (2015), 'I want to do anything which is decent and relates to my profession': Refugee doctors' and teachers' strategies of re-entering their professions in the UK. Journal of Refugee Studies, 28 (4), pp. 523-543.

Robjant K., Hassan R. \& Katona C. (2009), Mental health implications of detaining asylum seekers: systematic review. The British Journal of Psychiatry, 194 (4), pp. 306-312.

RöDER A. \& MüHLAu P. (2014), Are they acculturating? Europe's immigrants and gender egalitarianism. Social Forces, 92 (3), pp. 899-928.

Rosenberger S. \& König A. (2012), Welcoming the unwelcome: The politics of minimum reception standards for asylum seekers in Austria. Journal of Refugee Studies, 25 (4), pp. 537-554.

Ruiz I., Siegel M. \& VARgas-Silva C. (2015), Forced up or down? The impact of forced migration on social status. Journal of Refugee Studies, 28 (2), pp. 183-201.

Singh G. \& Clark B.D. (2013), Creating a frame: A spatial approach to random sampling of immigrant households in inner city Johannesburg. Journal of Refugee Studies, 26 (1), pp. 126-144.

Statistics Austia (2018, Bevölkerung nach Staatsangehörigkeit und Geburtsland [Population by nationality and country of birth]. Retrieved 24 October 2018, from $<$ http://www.statistik. at/web_de/statistiken/menschen_und_gesellschaft/bevoelkerung/bevoelkerungsstruktur/ bevoelkerung_nach_staatsangehoerigkeit_geburtsland/index.html>

Thомаs S.L. \& Thомаs S.D. (2004), Displacement and health. British Medical Bulletin, 69, pp. 115-127.

Turner S.W., Bowie C., Dunn G., et al. (2003), Mental health of Kosovan Albanian refugees in the UK. The British Journal of Psychiatry, 182 (5), pp. 444-448.

UDI. (2016a), Asylum applications lodged in Norway by citizenship and month (2015). from UDI (Norwegian Directorate of Immigration) retrieved from $<$ https://www.udi.no/en/statisticsand-analysis/statistics/asylsoknader-etter-statsborgerskap-og-maned-2015/>

UDI. (2016b), January - December 2015: Asylum applications lodged in Norway by Citizenship, Sex and Age. from UDI (Norwegian Directorate of Immigration), <https://www.udi.no/ en/statistics-and-analysis/statistics/asylum-applications-lodged-in-norway-by-citizenshipsex-and-age/>

UNHCR (2011), Asylum levels and trends in industrialized countries in 2010. Statistical overview of asylum applications lodged in Europe and selected non-European countries. Geneva, UNHCR.

UNHCR (2013a), A new beginning: Refugee integration in Europe. Geneva, The UN Refugee Agency.

UNHCR (2013b), Popoulation database. Retrieved 9 May 2018, from <http://www.unhcr. org/statistics/country/45c06c662/unher-statistical-online-population-database-sourcesmethods-data-considerations.html> 
ViKat A., Spéder Z., Beets G. et al. (2007), Generations and Gender Survey (GGS): Towards a better understanding of relationships and processes in the life course. Demographic Research, 17 (14), pp. 389-440.

Worbs S., Bund E. \& BöHm A. (2016), Asyl - und dann? Die Lebenssituation von Asylberechtigten und anerkannten Flüchtlingen in Deutschland [Asylum and then? The living situation of refugees in Germany]. Nürnberg, BAMF.

World Value Survey (2016), World Value Survey. Retrieved 27 January 2016, from < http://www. worldvaluessurvey.org/wvs.jsp.> 